\title{
Plasma natriuretic peptide levels reflect changes in heart failure symptoms, left ventricular size and function after surgical mitral valve repair
}

\author{
Harm H. H. Feringa · Don Poldermans · Patrick Klein · Jerry Braun • \\ Robert J. M. Klautz · Ron T. van Domburg • Arnoud van der Laarse • \\ Ernst E. van der Wall · Robert A. E. Dion · Jeroen J. Bax
}

Received: 7 June 2006/ Accepted: 15 July 2006/Published online: 29 August 2006

(C) Springer Science+Business Media B.V. 2006

\begin{abstract}
Background and aim N-terminal pro-B-type natriuretic peptide (NT-proBNP) has diagnostic and prognostic value in patients with heart failure. The present prospective study was designed to assess whether changes in NT-proBNP levels after surgical mitral valve repair reflect changes in heart failure symptoms and changes in left atrial size, left ventricular size and left ventricular function. Methods The study population consisted of 22 patients (mean age: $62.8 \pm 14.2$ years, $68 \%$ male) undergoing surgical mitral valve repair. Serial NT-proBNP measurements, transthoracic echocardiography and New York Heart Association (NYHA) class assessment were performed before and 6 months after surgery. Results All patients underwent successful mitral valve repair and no patients died during followup. The decrease in NT-proBNP level was associated with the reduction in left atrial dimension $(r=0.72, P<0.001)$, left ventricular end-systolic
\end{abstract}

H. H. H. Feringa $(\bowtie) \cdot$ P. Klein · J. Braun ·

R. J. M. Klautz · A. van der Laarse ·

E. E. van der Wall · R. A. E. Dion · J. J. Bax

Department of Cardiothoracic Surgery/Cardiology,

C-5, Leiden University Medical Center, Albinusdreef 2,

2333 ZA, Leiden, The Netherlands

e-mail: h.feringa@erasmusmc.nl

D. Poldermans · R. T. van Domburg

Cardiology, Erasmus Medical Center, Rotterdam,

The Netherlands dimension ( $r=0.63, P=0.002)$, left ventricular end-diastolic dimension $(r=0.46, P=0.031)$, and the increase in fractional shortening $(r=-0.63$, $P=0.002)$. Finally, patients with decreasing NTproBNP levels revealed a significant improvement in heart failure symptoms (NYHA class). Conclusion Changes in NT-proBNP after surgical mitral valve repair reflect changes in heart failure symptoms and changes in left atrial and ventricular dimensions and function.

Keywords Heart failure $\cdot$ Left ventricular function - Mitral valve repair ·

Natriuretic peptides

\section{Introduction}

The natriuretic peptides are endogenous cardiac hormones that include atrial natriuretic peptide (ANP), brain natriuretic peptide (BNP), and its amino-terminal portion $\mathrm{N}$-terminal pro-B-type natriuretic peptide (NT-proBNP) [1,2]. The BNP peptides are synthesized in the ventricular myocardium and released in response to ventricular wall stress $[3,4]$. In the clinical setting, both BNP and NT-proBNP have been demonstrated to provide important diagnostic and prognostic information in patients with heart failure [5-8].

Recently, elevated plasma BNP levels have been demonstrated in patients with chronic 
valvular disease [9-11]. In patients with chronic mitral regurgitation, the severity of regurgitation was directly related to the BNP levels [9-11]. The effect of mitral valve repair on BNP levels however, has not been studied. Accordingly, the topic of the current study was to evaluate the change in BNP levels after surgical correction of severe mitral regurgitation and to relate the findings to left ventricular reverse remodeling and improvement in clinical status after surgery.

\section{Material and methods}

\subsection{Study population}

Between July 2005 and September 2005, 22 consecutive patients undergoing mitral valve repair for severe mitral regurgitation were prospectively enrolled. All patients gave informed consent to participate in the study and the study was conducted in accordance with the Declaration of Helsinki. Patients with mitral valve stenosis (mitral valve area $<1.5 \mathrm{~cm}^{2}$ ) or aortic valve disease (severe aortic stenosis or regurgitation) were not included.

Mitral regurgitation was related to cardiomyopathy in 11 patients, and to degenerative disease in 11 patients.

\subsection{Assessment of symptoms and follow-up}

Clinical evaluation and assessment of symptoms using New York Heart Association (NYHA) class was conducted by the patient's referring cardiologist and was confirmed by an independent cardiothoracic surgeon at the time of hospital admission. A clinical follow-up was performed at 6 months after mitral valve repair to evaluate the change in NYHA class. During the 6-month follow-up period, adverse events including non-fatal myocardial infarction, repeat mitral valve surgery, cerebrovascular events, renal dysfunction and hospitalization for heart failure were noted.

\subsection{Echocardiography}

Prior to surgery, transthoracic echocardiography was performed in all patients. The patients were imaged in the left lateral decubitus position by using a commercially available system (Vingmed Vivid Seven, General Electric-Vingmed, Milwaukee, WI, USA). Using a $3.5 \mathrm{MHz}$ transducer, images were obtained at a depth of $16 \mathrm{~cm}$ in the parasternal (long- and short-axis images) and apical views (2- and 4-chamber images). From parasternal M-mode acquisitions, the left atrial diameter and left ventricular dimensions (end-systolic and end-diastolic diameter) were determined and the fractional shortening was calculated. The severity of mitral regurgitation was graded semi-quantitatively from color-flow Doppler in the conventional parasternal long-axis and apical 4-chamber images. Mitral regurgitation was characterized as mild $=1+($ jet area/left atrial area $<10 \%)$, moderate $=2+$ (jet area/left atrial area $10-20 \%)$, moderately severe $=3+$ (jet area/ left atrial area 20-45\%), and severe $=4+$ (jet area/left atrial area $>45 \%$ ) [12]. Immediately after surgery, transesophageal echocardiography was performed to assess residual mitral valve regurgitation. A transthoracic echocardiogram was repeated at 6 months follow-up to assess left atrial and ventricular dimensions, fractional shortening, the presence of residual mitral valve regurgitation, the transmitral diastolic gradient, the length of leaflet coaptation and the mitral valve area. Two experienced cardiologists who were blinded to the BNP levels and clinical data analyzed the echocardiographic data.

\subsection{NT-proBNP measurement}

Venous blood samples were collected on the day before and 6 months after surgery with the patient at rest and in semi-supine position. The samples were collected in chilled ethylene-diamine-tetra-acetic acid vacutainers and were immediately placed on ice. After centrifugation, the plasma samples were stored at $-80^{\circ} \mathrm{C}$ until assay. Plasma NT-proBNP concentrations was measured with an electrochemiluminescence immunoassay kit (Elecsys 2010, Roche GmbH, Mannheim, Germany). The method is a 'sandwich'-type quantitative immunoassay based on polyclonal antibodies against epitopes in the $\mathrm{N}$-terminal part of pro-BNP [13]. Assays were performed by a laboratory technician blinded to the patient's clinical data. 


\subsection{Statistical analysis}

The change in NT-proBNP levels from baseline to 6 months follow-up was calculated and expressed as percentage values. Changes in left atrial dimension, left ventricular end-systolic and end-diastolic dimensions and fractional shortening were also calculated and expressed as percentage values. Continuous data were expressed as mean $\left({ }_{ \pm} \mathrm{SD}\right)$ or median (interquartile range) when the distributions were skewed and compared using the Student $t$-test or the Mann-Whitney $U$ test when appropriate. Categorical data were compared using the Fisher's exact test. Group comparisons were performed with analysis of variance (ANOVA) techniques. The Pearson correlation coefficient was used to assess the association between changes in NT-proBNP levels and changes in echocardiographic variables. For all tests, a $P$ value $<0.05$ was considered significant. All analysis was performed using SPSS-11.0 statistical software (SPSS Inc., Chicago, Illinois).

\section{Results}

\subsection{Baseline characteristics}

The baseline characteristics of the 22 patients (mean age $62.8 \pm 14.2$ years, $68 \%$ male) are summarized in Table 1. Eight patients (36\%) were in NYHA class II, 5 (23\%) in class III and 9 $(41 \%)$ in class IV (mean NYHA class $3.1 \pm 0.9$ ). Prior to surgery, all patients presented with severe mitral valve regurgitation (grade 3-4+), with a mean regurgitation grade of $3.6 \pm 0.5$. Mean left atrial dimension was $4.9 \pm 0.7 \mathrm{~cm}$, mean left ventricular end-systolic dimension $4.5 \pm 0.8 \mathrm{~cm}$, mean left ventricular end-diastolic dimension $6.1 \pm 0.8 \mathrm{~cm}$ and mean fractional shortening $26.8 \pm 7.3 \%$. Median NT-proBNP level at baseline was $418 \mathrm{ng} / \mathrm{l}$ (interquartile range: 204$1258 \mathrm{ng} / \mathrm{l})$.

\subsection{Surgical results and follow-up}

Mean length of hospital stay was $9.8 \pm 4.3$ days. Transesophageal echocardiography immediately after surgery demonstrated competent valves with minimal residual mitral valve regurgitation in all patients (mitral regurgitation grade 0 in 13 patients, $59 \%$ and grade 1 in 9 patients, $41 \%$ ). All patients survived the 6-month follow-up period and no patients were lost to follow-up. During hospital stay and follow-up, none of the patients required repeat mitral valve surgery. Adverse events, including nonfatal myocardial infarction, cerebrovascular events, hospitalization for heart failure or endocarditis were not observed. Two patients $(9 \%)$ developed renal dysfunction in the postoperative period which was successfully treated with a short period of renal dialysis. None of the patients presented with renal dysfunction at 6 months follow-up. Median NT-proBNP level at 6 months follow-up was $426 \mathrm{ng} / \mathrm{l}$ (interquartile range $196-1172 \mathrm{ng} / \mathrm{l})$. In 10 patients (45\%), NTproBNP levels decreased $>10 \%$ and in 12 patients $(55 \%)$ NT-proBNP level remained unchanged or increased $>10 \%$ as compared to baseline values.

The patient population was subsequently divided into patients with a decrease in plasma NT-proBNP level versus patients with unchanged/ increased NT-proBNP plasma levels (Table 1). Baseline characteristics were comparable between the 2 groups, including baseline NT-proBNP levels. Only left atrial dimension was somewhat larger in patients with decreasing NT-proBNP levels after surgery as compared to those with increasing NT-proBNP levels $(P=0.04)$.

\subsection{Changes in NT-proBNP levels and symptoms}

NYHA class deteriorated in 3 patients (14\%), remained unchanged in $5(23 \%)$ and improved in $14(64 \%)$. The 10 patients with decreased NTproBNP levels exhibited a mean improvement in NYHA class of $2.0 \pm 1.1$, whereas the 12 patients with unchanged/increased NT-proBNP levels revealed a small but significant worsening in NYHA class $(0.3 \pm 0.9, P<0.001$ versus baseline $)$.

\subsection{Changes in NT-proBNP levels and echocardiographic variables}

The transthoracic echocardiogram at 6 months follow-up revealed a mean mitral regurgitation 
Table 1 Baseline clinical characteristics of the study population divided into patients with increasing and decreasing $\mathrm{N}$-terminal pro-B-type natriuretic peptide levels

$\begin{array}{llll}\text { Overall }(n=22) & \begin{array}{l}\text { Decreasing } \\ \text { NT-proBNP levels } \\ (n=10)\end{array} & \begin{array}{l}\text { Increasing } \\ \text { NT-proBNP levels } \\ (n=12)\end{array} & P \text { value } \\ \end{array}$

\begin{tabular}{|c|c|c|c|c|}
\hline \multicolumn{5}{|l|}{ Clinical variables } \\
\hline Age (years) & $62.8 \pm 14.2$ & $63.3 \pm 13.5$ & $62.4 \pm 15.3$ & 0.89 \\
\hline Male gender & $15(68.2)$ & $7(70.0)$ & $8(66.7)$ & 1.00 \\
\hline Hypertension & $4(18.2)$ & $3(30.0)$ & $1(8.3)$ & 0.29 \\
\hline Diabetes mellitus & $3(13.6)$ & $2(20.0)$ & $1(8.3)$ & 0.57 \\
\hline Chronic obstructive pulmonary disease & $4(18.2)$ & $1(10.0)$ & $3(25.0)$ & 0.59 \\
\hline Peripheral arterial disease & $3(13.6)$ & $3(30.0)$ & $0(0)$ & 0.078 \\
\hline History of stroke & $0(0)$ & $0(0)$ & $0(0)$ & - \\
\hline New York Heart Association class & $2.9 \pm 1.0$ & $3.3 \pm 0.9$ & $2.8 \pm 0.8$ & 0.23 \\
\hline \multicolumn{5}{|l|}{ Medication } \\
\hline Angiotensin-converting enzyme inhibitors & $10(45.5)$ & $6(60.0)$ & $4(33.3)$ & 0.39 \\
\hline Beta-blockers & $8(36.4)$ & $5(50.0)$ & $3(25.0)$ & 0.38 \\
\hline Diuretics & $9(40.9)$ & $4(40.0)$ & $5(41.7)$ & 1.00 \\
\hline \multicolumn{5}{|l|}{ Reason of mitral regurgitation } \\
\hline Degenerative disease & $11(50.0)$ & $5(50.0)$ & $6(50.0)$ & 1.00 \\
\hline Cardiomyopathy & $11(50.0)$ & $5(50.0)$ & $6(50.0)$ & 0.17 \\
\hline \multicolumn{5}{|l|}{ Echocardiographic measurements } \\
\hline Mitral regurgitation, grade & $3.6 \pm 0.5$ & $3.7 \pm 0.5$ & $3.6 \pm 0.5$ & 0.54 \\
\hline Left atrial dimension $(\mathrm{cm})$ & $4.9 \pm 0.7$ & $5.2 \pm 0.6$ & $4.7 \pm 0.7$ & 0.040 \\
\hline Left ventricular end-systolic dimension $(\mathrm{cm})$ & $4.5 \pm 0.8$ & $4.7 \pm 1.0$ & $4.3 \pm 0.6$ & 0.21 \\
\hline Left ventricular end-diastolic dimension $(\mathrm{cm})$ & $6.1 \pm 0.8$ & $6.3 \pm 0.7$ & $5.9 \pm 0.9$ & 0.27 \\
\hline Fractional shortening $(\%)$ & $26.8 \pm 7.3$ & $25.4 \pm 8.3$ & $28.0 \pm 6.6$ & 0.42 \\
\hline Baseline LN NT-proBNP level (ng/l) & $6.2 \pm 1.5$ & $6.6 \pm 1.4$ & $5.8 \pm 1.5$ & 0.18 \\
\hline
\end{tabular}

Values are expressed in mean \pm standard deviation or in number (\%). NT-proBNP denotes N-terminal pro-B-type natriuretic peptide

grade of $0.5 \pm 0.7$, mean length of leaflet coaptation of $0.9 \pm 0.2 \mathrm{~cm}$, mean mitral valve area of $2.6 \pm 0.9 \mathrm{~cm}^{2}$, and mean transmitral diastolic gradient of $3.3 \pm 1.2 \mathrm{mmHg}$. At follow-up, mean left atrial dimension was $4.4 \pm 0.5 \mathrm{~cm}$, mean left ventricular end-systolic dimension $4.2 \pm 0.9 \mathrm{~cm}$, mean left ventricular end-diastolic dimension $5.8 \pm 0.6 \mathrm{~cm}$, and mean fractional shortening $28.0 \pm 10.7 \%$. The 10 patients with decreased NTproBNP levels demonstrated significant reverse left ventricular remodeling, with a reduction in left ventricular end-systolic dimension from $4.7 \pm 1.0 \mathrm{~cm} \quad$ to $3.8 \pm 0.9 \mathrm{~cm} \quad(P=0.042), \quad$ a reduction in left ventricular end-diastolic dimension from $6.5 \pm 0.8 \mathrm{~cm}$ to $5.7 \pm 0.6 \mathrm{~cm}$ $(P=0.036)$ and a reduction in left atrial dimension from $5.3 \pm 0.6 \mathrm{~cm}$ to $4.3 \pm 0.5 \mathrm{~cm}$ $(P=0.001)$. Conversely, reverse left ventricular remodeling was not observed in the 12 patients with unchanged/increased NT-proBNP levels.
Mean left ventricular end-systolic dimension was $4.3 \pm 0.6 \mathrm{~cm}$ at baseline versus $4.5 \pm 0.7 \mathrm{~cm}$ $(P=0.43)$ at follow-up, and mean left ventricular end-diastolic dimension was $5.9 \pm 0.9 \mathrm{~cm}$ at baseline versus $5.8 \pm 0.6 \mathrm{~cm}$ at follow-up $(P=0.66)$. Mean left atrial dimension did also not change $(4.7 \pm 0.7 \mathrm{~cm}$ versus $4.5 \pm 0.6 \mathrm{~cm}$, $P=0.67)$. Scatter plots demonstrating the correlation between changes in NT-proBNP level and changes in echocardiographic variables during the 6-month follow-up period after mitral valve surgery are presented in Fig. 1. Decreases in NTproBNP levels at follow-up were significantly correlated with reductions in left atrial dimension $(r=0.72, P<0.001)$, left ventricular end-systolic dimension $(r=0.63, P=0.002)$, and left ventricular end-diastolic dimension $(r=0.46, P=0.031)$, indicating reverse remodeling; conversely, increases in NT-proBNP levels were related to increases in the different dimensions, indicating 
A

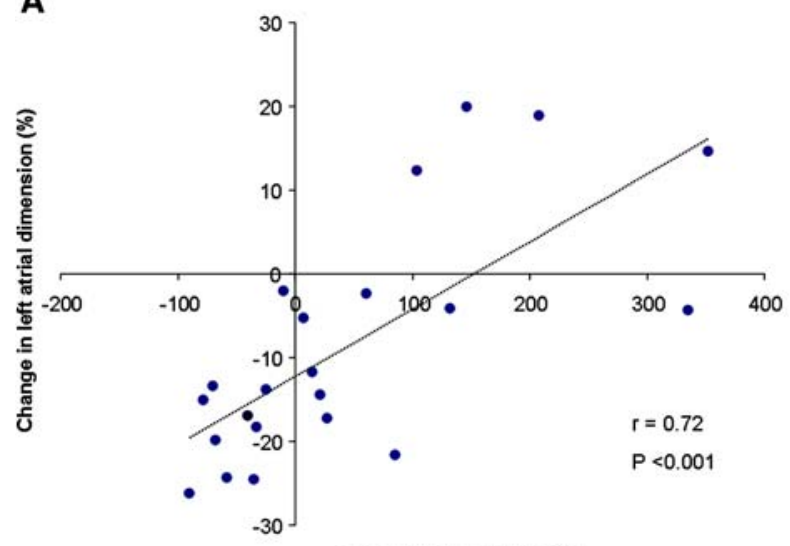

Change in NT-proBNP (\%)

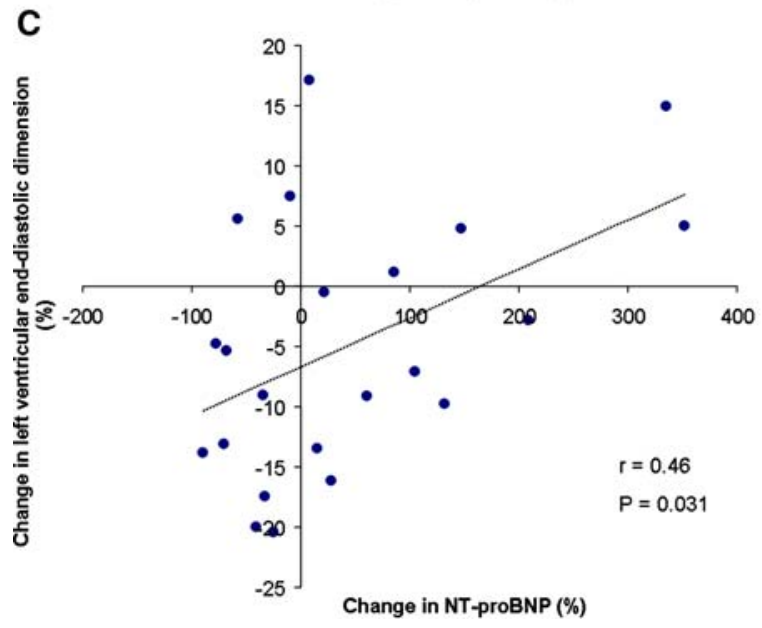

Fig. 1 Scatter plots demonstrating the correlation between changes in plasma N-terminal pro-B-type natriuretic peptide level (NT-proBNP) and changes in echocardiographic variables (A. left atrial dimension; B. left ventricular end-systolic dimension; C. left ventricular end-diastolic dimension; D. fractional shortening) during the 6-month follow-up period after mitral valve surgery. Of note, negative changes in left atrial dimension, left ventricular end-systolic dimension and left ventricular

ongoing dilatation. Moreover, decreases in NT-proBNP levels at follow-up were significantly correlated with improved fractional shorting ( $r=-0.63, P=0.002)$, indicating improved systolic function.

\section{Comments}

BNP has been used extensively in the diagnosis and prognosis of patients with heart failure [5-8]. More recently, Sutton and colleagues demon-

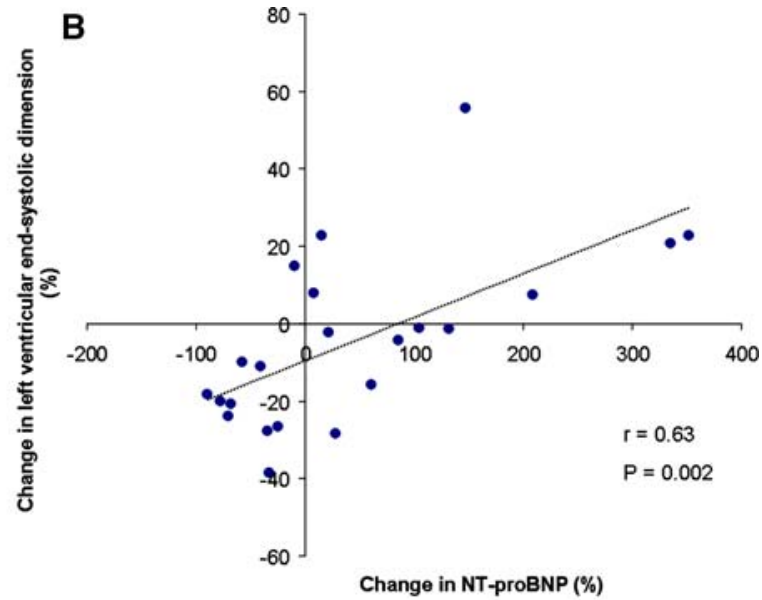

D

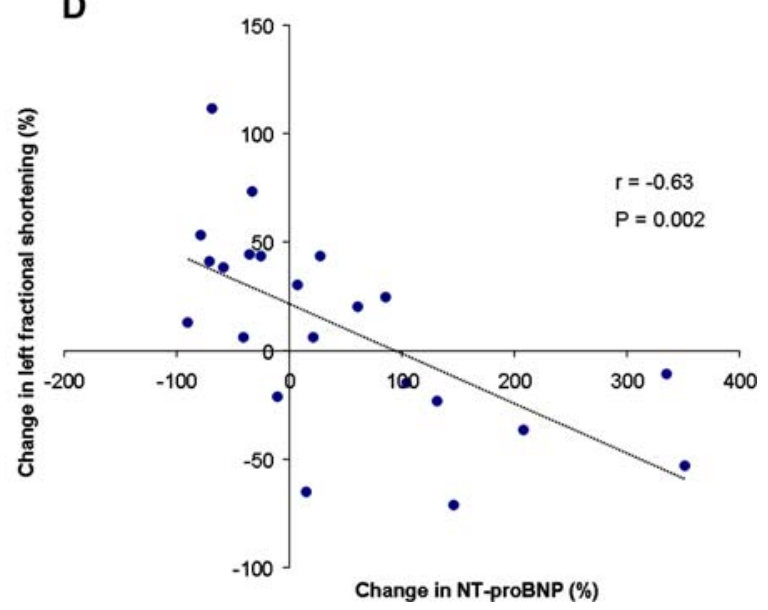

end-diastolic dimension indicate reductions in dimensions (reverse remodeling), whereas positive changes indicate ongoing dilatation. A positive change in fractional shortening indicates an increase in systolic function, whereas a negative change in fractional shortening indicates a decrease in systolic function. Negative changes in NT-proBNP indicate a reduction in plasma levels after surgery, whereas positive changes indicate an increase in plasma levels after surgery

strated in 49 patients with mitral regurgitation and preserved left ventricular ejection fraction that plasma levels of BNP and NT-proBNP levels were directly related to the severity of mitral valve regurgitation [11]. In addition, Detaint et al. evaluated 124 patients with chronic mitral regurgitation and demonstrated that BNP levels were correlated with long-term outcome [14]. In particular, higher BNP levels independently predicted mortality and the combined endpoint of mortality and heart failure. Moreover, the authors demonstrated that BNP levels in chronic mitral 
regurgitation were related to left atrial volumes, left ventricular end-systolic volume index, atrial fibrillation and heart failure symptoms. These findings indicate that the BNP plasma level reflects the hemodynamic, ventricular and atrial consequences of mitral regurgitation and that it may be a useful parameter to predict outcome in patients treated conservatively.

In the present study, NT-proBNP was assessed in patients with severe mitral regurgitation and re-assessed after surgical correction of regurgitation. The patient population consisted of patients with severe mitral valve regurgitation (grade 3-4+), left ventricular dilatation (left ventricular end-diastolic dimension $6.1 \pm 0.8 \mathrm{~cm}$ ) and reduced left ventricular function (fractional shortening $26.8 \pm 7.3 \%$ ), with a mean NYHA class of $3.1 \pm 0.9$.

All patients underwent successful surgical correction and demonstrated competent mitral valves without or minimal regurgitation on echocardiography at 6-months follow-up.

The current study is the first to report the association between NT-proBNP changes and echocardiographic outcome after surgical repair for mitral regurgitation. The changes in NTproBNP levels were directly related to changes in left atrial dimension, left ventricular dimensions and systolic function (as indicated by the fractional shortening, see Fig. 1).

In particular, the patients with a decrease in NT-proBNP levels exhibited a reduction in left atrial size, with reverse left ventricular remodeling. On the contrary, the patients with an increase in NT-proBNP levels did not exhibit this reverse remodeling and left atrial size did also not decrease after surgery. In addition, the patients with a reduction in NT-proBNP levels demonstrated an improvement in symptoms (reduction in NYHA class), whereas patients with an increase in NT-proBNP did not show a change in symptoms.

The findings confirm the use of NT-proBNP to assess clinical status. In addition to the heart failure population, NT-proBNP may be of use to reflect clinical status after mitral valve surgery, with a decrease in NT-proBNP indicating an improvement in symptoms and reverse left ventricular remodeling; conversely, an increase in
NT-proBNP should alert the clinician, since it was associated with absence of reverse remodeling and absence of improvement in symptoms.

The main limitations of the current study are the small sample size and the relatively short follow-up; accordingly, larger studies with longer follow-up are needed to confirm the present results. In addition, patients with mitral regurgitation with different etiologies were included, and findings need confirmation in homogenous populations. Also, the patients in the current study had moderate left ventricular dysfunction, and additional studies in patients with severe left ventricular dysfunction are needed.

\section{Conclusion}

In conclusion, changes in NT-proBNP after surgical mitral valve repair reflect changes in heart failure symptoms and changes in left ventricular dimensions. Changes in NT-proBNP levels may guide therapy after mitral valve surgery.

\section{References}

1. Levin ER, Gardner DG, Samson WK (1998) Natriuretic peptides. N Engl J Med 339:321-328

2. Hall C (2004) Essential biochemistry and physiology of (NT-pro)BNP. Eur J Heart Fail 6:257-260

3. Yoshimura M, Yasue H, Okumura K et al. (1993) Different secretion patterns of atrial natriuretic peptide and brain natriuretic peptide in patients with congestive heart failure. Circulation 87:464-469

4. Yasue H, Yoshimura M, Sumida H et al. (1994) Localization and mechanism of secretion of B-type natriuretic peptide in comparison with those of A-type natriuretic peptide in normal subjects and patients with heart failure. Circulation 90:195-203

5. Lainchbury JG, Campbell E, Frampton CM et al. (2003) Brain natriuretic peptide and N-terminal brain natriuretic peptide in the diagnosis of heart failure in patients with acute shortness of breath. J Am Coll Cardiol 42:728-735

6. Gardner RS, Ozalp F, Murday AJ et al. (2003) N-terminal pro-brain natriuretic peptide. A new gold standard in predicting mortality in patients with advanced heart failure. Eur Heart J 24:1735-1743

7. Bettencourt P, Azevedo A, Pimenta J et al. (2004) $\mathrm{N}$-terminal-pro-brain natriuretic peptide predicts outcome after hospital discharge in heart failure patients. Circulation 110:2168-2174

8. Nelson CA, Case C, McCrohon J et al. (2005) Relationship of extent and nature of dysfunctional 
myocardium to brain natriuretic peptide in patients with ischemic left ventricular dysfunction. Int $\mathbf{J}$ Cardiovasc Imaging 21:295-302

9. Mayer SA, De Lemos JA, Murphy SA et al. (2004) Comparison of B-type natriuretic peptide levels in patients with heart failure with versus without mitral regurgitation. Am J Cardiol 93:1002-1006

10. Brookes CI, Kemp MW, Hooper J et al. (1997) Plasma brain natriuretic peptide concentrations in patients with chronic mitral regurgitation. J Heart Valve Dis 6:608-612

11. Sutton TM, Stewart RA, Gerber IL et al. (2003) Plasma natriuretic peptide levels increase with symp- toms and severity of mitral regurgitation. J Am Coll Cardiol 41:2280-2287

12. Thomas JD (1997) How leaky is that mitral valve? Simplified Doppler methods to measure regurgitant orifice area. Circulation 95:548-555

13. Yeo KT, Wu AH, Apple FS et al. (2003) Multicenter evaluation of the Roche NT-proBNP assay and comparison to the Biosite Triage BNP assay. Clin Chim Acta 338:107-115

14. Detaint D, Messika-Zeitoun D, Avierinos JF et al. (2005) B-type natriuretic peptide in organic mitral regurgitation: determinants and impact on outcome. Circulation 111:2391-2397 